\title{
Asymptotic Free Energy of a System with Periodic Boundary Conditions
}

\author{
Michael E. Fisher \\ Baker Laboratory, Cornell University, Ithaca, New York \\ J. L. LEBOWITZ \\ Belfer Graduate School of Science, New York, New York
}

Received July 1, 1970

\begin{abstract}
A v-dimensional classical particle system in a torus, i.e., in a rectangular box with periodic boundary conditions, is considered in a canonical ensemble. Subject to mild restrictions over and above the usual stability and tempering conditions it is proved that the thermodynamic limit for the torus exists and is identical with that for systems contained in normal domains with boundaries or walls. If, in addition, the pair interaction potential $\varphi(\boldsymbol{r})$ decreases sufficiently rapidly (so that $r|\varphi(\boldsymbol{r})|$ is integrable at $\infty$ ), and satisfies some further regularity conditions, then the difference between the free energies of the torus and of the corresponding box is at most of the order of a surface term. Somewhat stronger results are indicated for the grand canonical pressure.
\end{abstract}

\section{Introduction}

The canonical free energy density of a system, namely

$$
F(\Omega) / V(\Omega)=-\beta g(\beta, \varrho ; \Omega),
$$

is calculated in statistical mechanics from the partition function according to the relation

$$
Z(\beta, N, \Omega)=e^{V(\Omega) g(\beta, \varrho ; \Omega)},
$$

where $\beta=1 / k_{\mathrm{B}} T$ measures the reciprocal temperature, and $N$ is the number of particles contained in the $v$-dimensional domain $\Omega$, of (generalized) volume $V(\Omega)$, which represents a physical container with hard impenetrable walls. The density is defined for integral $N$ by

$$
\varrho=N / V(\Omega),
$$

but the definition of $g(\beta, \varrho ; \Omega)$ may readily be extended to general values of $\varrho$ by linear interpolation (see Ref. [1]). We assume a particle Hamiltonian of the standard form

$$
\mathscr{H}_{N}=\sum_{i=1}^{N} p_{i}^{2} / 2 m+U_{N}\left(\boldsymbol{r}_{1}, \ldots \boldsymbol{r}_{n}\right),
$$


where the $\boldsymbol{p}_{i}$ and $\boldsymbol{r}_{i}=\left(r_{i, 1}, r_{l, 2}, \ldots r_{l, v}\right)$ are the momentum and coordinate vectors of the $i$ th particle and $U_{N}$ is the total interaction energy. Our considerations will extend straightforwardly to systems with a finite number of different species of particle but we omit this complication in the interests of simplicity.

For a classical system one has

$$
Z(\beta, N, \Omega)=\Lambda_{T}^{-v N} Q(\beta, N, \Omega)
$$

where the thermal De Broglie wavelength is, as usual,

$$
\Lambda_{T}=\left(h^{2} / 2 \pi m k_{\mathrm{B}} T\right)^{\frac{1}{2}},
$$

while the configuration integral is

$$
Q(\beta, N, \Omega)=\frac{1}{N !} \int_{\Omega} \cdots \int_{\Omega} \exp \left[-\beta U_{N}\left(\boldsymbol{r}_{1}, \ldots \boldsymbol{r}_{N}\right)\right] d^{v} \boldsymbol{r}_{1}, \ldots d^{v} \boldsymbol{r}_{N} .
$$

Recently the asymptotic properties of the free energy density $g(\beta, \varrho ; \Omega)$ have been discussed [1-3] in the thermodynamic limit in which the volumes $V_{k}=V\left(\Omega_{k}\right)$ of an infinite sequence of domains $\Omega_{k}$ increase without limit (while the corresponding densities $\varrho_{k}$ approach a finite limit $\varrho$, or, alternatively the interpolated density $\varrho$ remains fixed). Provided the potentials satisfy both a stability condition, namely [1-3]

A Stability

$$
\begin{array}{ll} 
& U_{N}\left(\boldsymbol{r}_{1}, \ldots \boldsymbol{r}_{N}\right) \geqq-N w_{A} \\
\text { for all } & \boldsymbol{r}_{1}, \ldots \boldsymbol{r}_{N} \text { and all } N\left(w_{A}<\infty\right) ;
\end{array}
$$

and $\mathbf{B}$, a suitable tempering condition (see below), and provided that the shapes of the domains $\Omega_{k}$ remain sufficiently regular as $k \rightarrow \infty$ (principally the "surface-to-volume" ratio must go to zero) $[1,3]$ it has been proved rigorously that the limit

$$
\lim _{k \rightarrow \infty} g\left(\beta, \varrho ; \Omega_{k}\right)=g_{\infty}(\beta, \varrho),
$$

exists. Furthermore, the limiting free energy function, $g_{\infty}(\beta, \varrho)$, is unique being, in particular, independent of the details of the shapes of the $\Omega_{k}$. This result justifies the relationship between thermodynamics and statistical mechanics normally postulated for systems in "realistic" containers. For theoretical and numerical purposes, however, it is often very convenient to suppose that the system is confined to a torus $\Pi$; that is to a rectangular domain or box $\Lambda$ of sides $\boldsymbol{L}=\left(L_{1}, \ldots L_{v}\right)$, so that

$$
\boldsymbol{r} \subset \Lambda \Leftrightarrow 0<r_{i, \alpha} \leqq L_{\alpha} \quad(\alpha=1,2, \ldots \nu),
$$


on which periodic boundary conditions are imposed. We defer a precise specification of the meaning of the term periodic boundary conditions to Section II.

The main practical advantage of periodic boundary conditions is that they make all the properties of the system translationally invariant (on the torus). This eliminates explicit surface effects. It is hence expected that a 'periodic system' or torus $\Pi$ with a limited number of particles, $N$, will better approximate the properties of an infinite thermodynamic system than a system of $N$ particles in a rigid walled container, $\Lambda$. Nearly all machine computations are thus carried out on periodic systems.

Naturally it is a common belief that the free energy calculated for a torus with periodic boundary conditions will, in the thermodynamic limit

$$
\boldsymbol{L} \rightarrow \infty \quad \text { i.e., } \quad L_{\alpha} \rightarrow \infty \quad(\text { all } \alpha),
$$

be identical with that calculated in the usual way for a sequence of 'normal domains' with walls or boundaries. This has not, however, been proved nor, as far as we know, is it an obvious corollary of existing work. In this article we supply the missing proof by demonstrating that the canonical free energy of a torus, $\Pi$, is indeed asymptotically, the same as that of the corresponding box $\Lambda$ (or other regular domain $\Omega$ ). Our proof applies to classical systems with only minor restrictions over and above those, like stability, normally required for the existence of the thermodynamic limit (see Section II) [1-3]. With minor modifications it applies also to a partial torus in which periodic boundary conditions are imposed on $\Lambda$ in fewer than $v$ directions. Furthermore, there is no difficulty in extending the proof to the grand canonical ensemble. On the other hand, we have not overcome all the difficulties associated with quantum-mechanical systems nor have we considered the fundamental cases of Coulomb [4] or dipolar forces [5] for which further special considerations are essential.

The proof commences after Section II in which the explicit meaning of periodic boundary conditions is examined and where the stability, tempering and shape conditions used in the proof are stated. The first part of the proof, in Section III, is the calculation of an asymptotic lower bound to the free energy density, $g(\beta, \varrho ; \Pi)$, of the torus in terms of the standard limiting free energy density $g_{\infty}(\beta, \varrho)$. This step is achieved along lines following closely the existing proofs $[1,2]$ of the thermodynamic limit in a normal system: it involves "cutting" the torus and "squeezing" the box $\Lambda$ into a smaller box $\Lambda^{-}$which is easily shown to reduce the partition function except for an asymptotically negligible term which arises from longrange repulsive interactions. The second step of the proof, in Section IV, entails the calculation of an asymptotic upper $19 *$ 
bound. This is more tricky: again the torus is cut but the maximum possible loss of attractive (negative) potential energy by interactions across the dividing surface must now be estimated and shown to be asymptotically negligible. Combination of the upper and lower bounds establishes the basic result:

Theorem. Subject to the conditions stated in Section II the free energy density of a classical particle system in a torus $\Pi$, i.e., with periodic boundary conditions, satisfies

$$
\lim _{\boldsymbol{L} \rightarrow \infty} g(\beta, \varrho ; \Pi)=g_{\infty}(\beta, \varrho) .
$$

where $g_{\infty}(\beta, \varrho)$ is the limiting free energy density for normal domains defined in (1.9).

Some remarks concerning quantum-mechanical systems are made at the end of Section III.

Finally, in Section $\mathrm{V}$ we demonstrate that when the pair interactions decay sufficiently rapidly [essentially so that $r|\varphi(\boldsymbol{r})|$ is integrable for large $r$ ], the difference between the free energy of a finite torus and that of the corresponding normal box is at most a "surface" term. Our analysis here for the lower bound to $g(\Pi)-g(\Lambda)$ is restricted to strongly tempered potentials (nonpositive for $r>R_{0}$ ) without a hard core. We indicate, however, in Section VI how, in the grand canonical ensemble, the corresponding result can also be derived for (suitable) weakly tempered potentials with a hard core. In addition, we show that the grand canonical pressure for both box $\Lambda$ and torus $\Pi$ differ from the thermodynamical limiting pressure by no more than a surface-to-volume term. We may comment at this point that it is much harder to prove that $g(\Pi)-g(\Lambda)$ and $g(\Lambda)-g_{\infty}$, etc. vary asymptotically precisely as surface terms. Indeed although this is the normal thermodynamic expectation, and has been verified for certain models [6] it may not always be true. In any event we do no more in this paper than raise the question.

\section{Periodicity, Stability and Tempering}

Before discussing the various stability, tempering and shape conditions we will employ in the proof, we make precise the implication of the phrase "periodic boundary conditions are imposed on the box $\Lambda$ ". Its meaning may be stated as follows:

(a) The particles are supposed to interact only through a pair interaction potential $\varphi(\boldsymbol{r})$, so that (with $\boldsymbol{r}_{i}$ in $\Lambda$ ) we have

$$
U_{N}=U_{N}\{\varphi\}=\frac{1}{2} \sum_{i=1}^{N} \sum_{j \neq 1}^{N} \varphi\left(\boldsymbol{r}_{j}-\boldsymbol{r}_{i}\right) ;
$$


(b) in terms of $v$ orthogonal unit vectors $\boldsymbol{e}_{\alpha}$, the lattice vectors

$$
\boldsymbol{l}_{\boldsymbol{t}}=\sum_{\alpha=1}^{v} t_{\alpha} L_{\alpha} \boldsymbol{e}_{\alpha}, \quad \boldsymbol{t}=\left(t_{1}, \ldots t_{v}\right),
$$

are defined, where the $t_{i}$ are positive or negative integers or zero; the integer vectors $t$ index the cells $\Lambda_{t}$ congruent to $A \equiv \Lambda_{0}$ into which space may thus be divided;

(c) the periodized Hamiltonian $\mathscr{H}_{N}^{\Pi}$ is defined by (1.4) except that the periodized potential energy, $U_{N}^{I}\{\varphi\}$, is calculated by extending the summation on $j$ in (2.1) to run over all the images, $\boldsymbol{r}_{j}+\boldsymbol{l}_{\boldsymbol{t}}$, of the particles at $r_{j}$ as periodically repeated in each cell throughout space. The integrations on each $\boldsymbol{r}_{i}$ in (1.7) run over the full domain $\Lambda$.

Explicitly this construction yields (with $\boldsymbol{r}_{i}$ in $\Lambda$ )

$$
U_{N}^{\Pi}\{\varphi\}=U_{N}^{\Pi}\left(\boldsymbol{L} ; \boldsymbol{r}_{1}, \ldots \boldsymbol{r}_{N}\right)=\frac{1}{2} \sum_{i=1}^{N} \sum_{j=1}^{N} \sum_{\boldsymbol{t}}^{\prime} \varphi\left(\boldsymbol{r}_{j}+\boldsymbol{l}_{\boldsymbol{t}}-\boldsymbol{r}_{i}\right),
$$

where the prime on the summation over $t$ indicates that when $i=j$ the term $\boldsymbol{t}=0$ [corresponding to the self-interaction, $\varphi(\mathbf{0})]$ is omitted. The definition (2.3) may also be expressed as

$$
U_{N}^{\Pi}\{\varphi\}=U_{N}\left\{\varphi^{\Pi}\right\}+N u_{0}^{\Pi}\{\varphi\}, \quad\left(\boldsymbol{r}_{i} \subset \Lambda\right),
$$

where the periodized pair potential is

$$
\varphi^{\Pi}(\boldsymbol{r})=\sum_{\boldsymbol{t}} \varphi\left(\boldsymbol{r}+\boldsymbol{l}_{\boldsymbol{t}}\right),
$$

and the periodized self-interaction potential is

$$
u_{0}^{\Pi}\{\varphi\}=\frac{1}{2} \sum_{\boldsymbol{t} \neq 0} \varphi\left(\boldsymbol{l}_{\boldsymbol{t}}\right) .
$$

The periodic partition function is, of course, calculated from (1.5) to (1.7) with $\Omega \equiv \Lambda$ and with $U_{N}^{M}$ replacing $U_{N}$.

Naturally the question of the convergence of the infinite lattice sums in (2.3), (2.5), and (2.6) must be considered. As regards the positive or "repulsive" parts of the potential this is ensured by the

B' Mild Tempering Condition

$$
\varphi(\boldsymbol{r}) \leqq|\boldsymbol{r}|^{-v} \theta^{+}(|\boldsymbol{r}|) \text { for }|\boldsymbol{r}| \geqq r_{0}>0
$$

where $r^{-v} \theta^{+}(r)$ is a continuous monotonic nonincreasing function for which

$$
\int_{r_{0}}^{\infty} r^{-1} \theta^{+}(r) d r<\infty
$$


A condition of this sort is already needed for the proof of the existence of the normal thermodynamic limit [1,3]. (Actually the condition $\mathbf{B}^{\prime}$ is slightly less restrictive than the weak tempering condition, $\mathbf{B}$, introduced originally in Ref. [1], but the original arguments can quite easily be modified to utilize only $\mathbf{B}^{\prime}$.) Evidently the restriction (2.8) rules out Coulomb [4] and dipolar forces [5] but, as mentioned, these require further special conditions in any case (e.g., asymptotic electroneutrality must be imposed).

To insure the convergence of the lattice sums with respect to the negative or "attractive" parts of the potential we will impose the corresponding

\section{A' Partial Stability Condition}

$$
\varphi(\boldsymbol{r}) \geqq-|\boldsymbol{r}|^{-v} \theta^{-}(|\boldsymbol{r}|), \quad|\boldsymbol{r}| \geqq 0,
$$

where $r^{-v} \theta^{-}(r)$ is a monotonic nonincreasing functions for which

$$
\int_{0}^{\infty} r^{-1} \theta^{-}(r) d r<\infty
$$

We call this a partial stability condition since, in conjunction with the condition that $\varphi(\boldsymbol{r})$ has a repulsive hard core [i.e., $\varphi(\boldsymbol{r})=+\infty$ for $|\boldsymbol{r}| \leqq a>0]$, it is sufficient to establish stability for both $U_{N}\{\varphi\}$ and for $U_{N}^{\Pi}\{\varphi\}[1,3]$. We also point out that in the case of strictly short range potentials $[\varphi(\boldsymbol{r}) \equiv 0$ for $|\boldsymbol{r}|$ exceeding some $b<\infty]$ it is not hard to prove from (2.3) that the stability of $U_{N}\{\varphi\}$ implies the stability of $U_{N}^{I I}\{\varphi\}$ with the same constant [7]. Before discussing the stability of the periodized potentials more generally, however, we re-express them once more.

When the potential $\varphi(\boldsymbol{r})$ is everywhere bounded and has a Fourier transform

$$
\hat{\varphi}(\boldsymbol{k})=\int d^{v} \boldsymbol{r} e^{-i \boldsymbol{k} \cdot \boldsymbol{r}} \varphi(\boldsymbol{r}),
$$

the standard and periodized total potential energies (2.1) and (2.3) may be written in the comparable forms

$$
U_{N}\{\varphi\}=\frac{1}{2} \int d^{v} \boldsymbol{k} \hat{\varphi}(\boldsymbol{k})|n(\boldsymbol{k})|^{2}-\frac{1}{2} N \varphi(\mathbf{0})
$$

where

$$
\hat{n}(\boldsymbol{k})=\sum_{i=1}^{N} e^{-i \boldsymbol{k} \cdot \boldsymbol{r}_{i}}=n(-\boldsymbol{k})^{*}
$$

and

$$
U_{N}^{I I}\{\varphi\}=\frac{1}{2}\left[\prod_{\alpha=1}^{v}\left(2 \pi / L_{\alpha}\right)\right] \sum_{\boldsymbol{q}} \hat{\varphi}\left(\boldsymbol{k}_{\boldsymbol{q}}\right)\left|\hat{n}\left(\boldsymbol{k}_{\boldsymbol{q}}\right)\right|^{2}-\frac{1}{2} N \varphi(\mathbf{0})
$$


where the discrete reciprocal lattice vectors, $\boldsymbol{k}_{\boldsymbol{q}}$, are defined by

$$
\begin{aligned}
\boldsymbol{k}_{\boldsymbol{q}} & =\left(2 \pi q_{1} / L_{1}, \ldots 2 \pi q_{v} / L_{v}\right), \\
\boldsymbol{q} & =\left(q_{1}, \ldots q_{v}\right), \quad q_{\alpha}=0, \pm 1, \pm 2, \ldots .
\end{aligned}
$$

In practice it is frequently through the implicit or explicit use of the formula (2.14) that periodic boundary are introduced.

Now in order to satisfy the stability condition $\mathbf{A}$, without requiring hard cores, a very useful auxiliary condition has been introduced $[1,3]$, namely,

Ab Positive plus positive type potentials

$$
\varphi(\boldsymbol{r}) \geqq \varphi_{+}(\boldsymbol{r})=\int d \boldsymbol{k} e^{i \boldsymbol{k} \cdot \boldsymbol{r}} \varphi_{+}(\boldsymbol{k})
$$

where $\varphi_{+}(\boldsymbol{r})$ is continuous and integrable and

$$
\hat{\varphi}_{+}(\boldsymbol{k}) \geqq 0 \text {. }
$$

That (2.16) and (2.17) imply stability for $U_{N}\{\varphi\}$ can be seen directly from (2.12). It is equally clear from (2.14), however, that condition $\mathbf{A} \mathbf{b}$ also insures stability for the periodized potential $U_{N}^{I I}\{\varphi\}$. For this reason we will consider only potentials satisfying $\mathbf{A} \mathbf{b}$. This is a very wide class of potentials (see Refs. $[1,3,8]$ ) and, indeed, no stable potentials are presently known for $v>1$ which do not satisfy $\mathbf{A b}$. In particular, if the potential satisfies the partial stability condition $\mathbf{A}^{\prime}$ and diverges positively at the origin faster than $1 / r^{v+\varepsilon}(\varepsilon>0)$, then it also satisfies $\mathbf{A} \mathbf{b}$ and is stable $[1,3,8]$.

To complete the second part of the proof [upper bound for $g(\beta, \varrho ; \Pi)$ ] we actually need a somewhat stronger stability condition, at least as regards the total periodized potential $U_{N}^{I I}\{\varphi\}$. To state this we decompose the pair potential into positive and negative parts:

$$
\begin{array}{ll} 
& \varphi(\boldsymbol{r})=\varphi_{>}(\boldsymbol{r})+\varphi_{<}(\boldsymbol{r}), \\
\text { where } \quad & \varphi_{>}(\boldsymbol{r}) \geqq 0 \text { and } \varphi_{<}(\boldsymbol{r}) \leqq 0,
\end{array}
$$

and define the extended potential

$$
\varphi(\eta ; \boldsymbol{r})=\varphi(\boldsymbol{r})+\eta \varphi_{<}(\boldsymbol{r}) .
$$

In the extended pair potential the attractive (negative) parts are thus multipled by a factor $(1+\eta)$. We will then require:

$\mathbf{A}^{I I} \boldsymbol{\eta}$ Extended Periodic Stability

For some $\eta_{0}>0$ and all $N$

$$
U_{N}^{I I}(\eta)=U_{N}^{I I}\left\{\varphi(\boldsymbol{r})+\eta \varphi_{<}(\boldsymbol{r})\right\} \geqq-N w_{A}(\eta),
$$

with $w_{A}(\eta)<\infty$ for $\eta \leqq \eta_{0}$. 
By the previous arguments this condition will be satisfied if the extended potential $\varphi(\eta ; \boldsymbol{r})$ satisfies condition $\mathbf{A} \mathbf{b}$ for $\eta \leqq \eta_{0}$. It will always be satisfied if $\varphi$ consists of an infinite hard core plus a tempered potential. Furthermore, since the stability of $U_{N}^{\Pi}\left(\eta_{1}\right)$ clearly implies the stability of $U_{N}^{\Pi}(\eta)$ for all $\eta \leqq \eta_{1}$ this condition only serves to exclude certain pathological "borderline" potentials that are just on the edge of being catastropic [8].

Finally, we will employ a shape condition on the sequence of tori used in taking the thermodynamic limit. If

$$
\tilde{L}=\min _{\alpha}\left\{L_{\alpha}\right\}
$$

is the shortest side of the corresponding box $\Lambda$, we will require

D* Uniform Regularity

$$
V(\Lambda) / \tilde{L}^{v}=\prod_{\alpha=1}^{v}\left(L_{\alpha} / \tilde{L}\right) \leqq c_{1}
$$

where $c_{1}<\infty$ is fixed independent of $\Lambda$.

This is a special case of the more general uniform regularity shape condition introduced in Ref. [1] for systems with weakly tempered potentials. (It is not needed if the potentials are of strictly short range.)

\section{Lower Bound}

To obtain a lower bound to the periodic free energy density $g(\beta, \varrho ; \Pi)$ we compare the torus $\Pi$ with a reduced box $\Lambda^{-}$of sides $L_{\alpha}-R$. Equivalently, in the periodically repeated picture, we cut channels of width $R$ between the cells $\Lambda_{t}$ and discard all interactions between the reduced cells $\Lambda_{t}^{-}$so formed. (Of course, we suppose that all particles are now confined to the reduced cells.)

For simplicity in the first instance let us suppose the potentials satisfy [1] the

\section{B* Strong Tempering Condition}

$$
\varphi(\boldsymbol{r}) \leqq 0 \text { for }|\boldsymbol{r}|>R_{0} .
$$

The reduction of the domain of integration in the configurational integral $Q(\beta, N, \Pi)$, from $\Lambda$ to $\Lambda^{-}$must decrease its magnitude. Furthermore, if we take $R$ fixed but exceeding $R_{0}$, the residual cell-cell interactions across the channels are all attractive, i.e., nonpositive, so that discarding them can only reduce, or leave unchanged, the integral $Q\left(\beta, N, \Lambda^{-}\right)$. It follows that

$$
Z(\beta, N, \Pi) \geqq Z\left(\beta, N, \Lambda^{-}\right)
$$


and so, by (1.2),

$$
g(\beta, \varrho ; \Pi) \geqq\left(\varrho / \varrho^{-}\right) g\left(\beta, \varrho^{-} ; \Lambda^{-}\right),
$$

where the new density is

$$
\varrho^{-}=\varrho V(\Lambda) / V\left(\Lambda^{-}\right)=\varrho \prod_{\alpha=1}^{v}\left[1-\left(R / L_{\alpha}\right)\right]^{-1} .
$$

For the bound (3.3) to be meaningful we must insist that $\varrho^{-}<\varrho_{\max }\left(\Lambda^{-}\right)$, where the maximum density is determined by any hard core present in $\varphi(\boldsymbol{r})$. In the thermodynamic limit $(1.11)$ we have [1-3] $\varrho_{\max }(\Lambda) \rightarrow \varrho_{\max }^{\infty}$ and since we also will have $R / L_{\alpha} \rightarrow 0$ and $\varrho^{-} \rightarrow \varrho$ this restriction on the density is asymptotically harmless. (In the arguments below we will allow $R \rightarrow \infty$ but always ensure that $R / L_{\infty} \rightarrow 0$; thus the restriction will again be harmless and, hence, it will not be mentioned explicitly.)

Now if we take the thermodynamic limit in the inequality (3.3) and recall the continuity of the limit function (see Refs. [1-3]) we obtain

$$
\liminf _{\boldsymbol{L} \rightarrow \infty} g(\beta, \varrho ; \Pi) \geqq g_{\infty}(\beta, \varrho),
$$

which is the desired asymptotic lower bound [see (1.9)].

More generally we may consider potentials satisfying the mild tempering condition $\mathbf{B}^{\prime}$, stated in Section II, namely,

$$
\varphi(\boldsymbol{r}) \leqq|\boldsymbol{r}|^{-v} \theta^{+}(|\boldsymbol{r}|) \text { for }|\boldsymbol{r}|>r_{0}
$$

where $r^{-v} \theta^{+}(r)$ is continuous, monotonic nonincreasing and $r^{-1} \theta^{+}(r)$ is integrable on $\left(r_{0}, \infty\right)$. Now from the definition (2.3) of $U_{N}^{I I}$ the total cellcell interaction energy is clearly

$$
\Phi_{N}\left(\boldsymbol{L} ; \boldsymbol{r}_{1} \ldots \boldsymbol{r}_{N}\right)=\frac{1}{2} \sum_{i=1}^{N} \sum_{j=1}^{N} \sum_{\boldsymbol{t} \neq \mathbf{0}} \varphi\left(\boldsymbol{r}_{j}+\boldsymbol{l}_{t}-\boldsymbol{r}_{i}\right) .
$$

If the distance between a particle $\boldsymbol{r}_{i}$ and the nearest image of another particle is not less than $R \geqq r_{0}$, as is the case when the particles are confined to the reduced box $\Lambda^{-}$. We have from (3.6)

$$
\begin{aligned}
\Phi_{N}(\boldsymbol{L}, R) & \leqq \frac{1}{2} \sum_{i=1}^{N} \sum_{j=1}^{N} \sum_{\boldsymbol{t} \neq 0}\left|\boldsymbol{r}_{j}+\boldsymbol{l}_{j}-\boldsymbol{r}_{i}\right|^{-v} \theta^{+}\left(\left|\boldsymbol{r}_{j}+\boldsymbol{l}_{j}-\boldsymbol{r}_{i}\right|\right) \\
& \leqq \frac{1}{2} N^{2} \sum_{\boldsymbol{t} \neq 0} \tilde{R}_{\boldsymbol{t}}^{-v} \theta^{+}\left(\tilde{R}_{\boldsymbol{t}}\right),
\end{aligned}
$$

where $\tilde{R}_{t}$ is the least distance between a point in cell $\Lambda_{0}^{-}$and one in $\Lambda_{t}^{-}$ with $\boldsymbol{t} \neq 0$. Following Lebowitz and Penrose [9] we estimate the lattice sum by grouping the cells into shells. In the first shell there are $3^{v}-1$ 
cells and $\tilde{R}_{t} \geqq R ;$ in the second shell there are $5^{v}-3^{v}$ cells and $\tilde{R}_{t} \geqq R$ $+\tilde{L}>2 R$, where, as before,

$$
\tilde{L}=\min _{\alpha}\left\{L_{\alpha}\right\},
$$

and we naturally assume $\tilde{L}>R$. In the $s$ th shell there are $(2 s+1)^{v}$ $-(2 s-1)^{v}$ cells and $\tilde{R}_{t}>s R$. From $(2.8)$ we hence obtain

where

$$
\Phi_{N}(\boldsymbol{L}, R) \leqq \frac{1}{2} N^{2} R^{-v} \psi^{+}(R),
$$

$$
\psi^{+}(R)=\sum_{s=1}^{\infty}\left[\left(2+s^{-1}\right)^{v}-\left(2-s^{-1}\right)^{v}\right] \theta^{+}(s R) .
$$

Since $r^{-1} \theta^{+}(r)$ is integrable, $s^{-1} \theta^{+}(s R)$ is summable on $s$. Furthermore, since $\theta^{+}(s R)$ vanishes continuously as $R \rightarrow \infty$ we conclude that $\psi^{+}(R)$ is continuous and that

$$
\psi^{+}(R) \rightarrow 0 \quad \text { as } \quad R \rightarrow \infty .
$$

On returning to the previous argument comparing $\Pi$ with $\Lambda^{-}$but allowing now for the loss of the cell-cell interactions $\Phi_{N}(L, R)$, we obtain in place of (3.3), the inequality

$$
g(\beta, \varrho ; \Pi) \geqq\left(\varrho / \varrho^{-}\right) g\left(\beta, \varrho^{-} ; \Lambda^{-}\right)-\frac{1}{2} \beta \varrho^{2} V(\Lambda) R^{-v} \psi^{+}(R),
$$

where $\varrho^{-}$is still defined by (3.4). Now by (3.11) and (3.12) the function $1 / \psi^{+}(R)$ is continuous and diverges to $+\infty$ as $R \rightarrow \infty$. Accordingly for large enough $\tilde{L}=L_{\min }$ we may choose $R=R(L)$ so that

$$
R^{v} /\left[\psi^{+}(R)\right]^{1-\varepsilon}=\tilde{L}^{v}, \quad 1>\varepsilon>0 .
$$

This choice implies

$$
\text { (i) } \quad R \rightarrow \infty \text {, and (ii) } R / \tilde{L} \rightarrow 0 \text { as } \boldsymbol{L} \rightarrow \infty \text {. }
$$

By (2.4) the relation (ii) insures that $\varrho^{-} \rightarrow \varrho$ as $L \rightarrow \infty$ which is clearly essential. Then if we use the shape condition $\mathbf{D}^{*}$ stated in Section II [Eq. (2.22)], and make the choice (3.14), we find

$$
\begin{aligned}
V(\Lambda) R^{-v} \psi^{+}(R) & \leqq c_{1}(\tilde{L} / R)^{v} \psi^{+}(R), \\
& \leqq c_{1}\left[\psi^{+}(R)\right]^{\varepsilon} \rightarrow 0 \text { as } \quad L \rightarrow \infty .
\end{aligned}
$$

With the above choice of $R$ we can thus take the limit in the inequality (3.13) to obtain, again, the required lower bound (3.5).

Finally, we remark that this lower bound can be derived equally well for a quantum-mechanical system obeying any statistics. The main point is that after cutting the channels one must also insist that the wave functions vanish on the boundaries of the reduced box $\Lambda^{-}$. (In the torus $\Pi$ 
only continuity of the wave functions is required.) This boundary condition increases the kinetic energy and can only reduce the partition function further (see Refs. [1,2,10)]. Thus in this respect quantummechanical periodic boundary conditions cause no difficulty. More generally, however, changing the boundary conditions from the customary vanishing boundary conditions $[1-3]\left[\right.$ i.e., $\Psi_{N}\left(\boldsymbol{r}_{1}, \ldots \boldsymbol{r}_{N}\right) \equiv 0$ for $\boldsymbol{r}_{i}$ on the boundary of $\Omega$ ], to normal derivative conditions [i.e., $\boldsymbol{n} \cdot \nabla \Psi_{N} \equiv 0$ on the boundary], or to intermediate or periodic conditions introduces complications even for systems without periodic potentials. These questions have been considered by Lieb [10], Robinson [11] and, very recently, by Gallavotti et al. [12]. The results of these last authors should enable one to extend the remainder of our discussion of the torus to the quantum-mechanical case.

\section{Upper Bound}

To obtain an upper bound for $g(\beta, \varrho ; \Pi)$ we compare it with $g(\beta, \varrho ; \Lambda)$ and try to obtain a lower bound on the attractive part of the cell-cell interactions lost in going from $\Pi$ to $\Lambda$. To avoid difficulties with any hard cores or positively unbounded pieces of $\varphi(\boldsymbol{r})$ we use the decomposition

$$
\begin{gathered}
\varphi(\boldsymbol{r})=\varphi_{>}(\boldsymbol{r})+\varphi_{<}(\boldsymbol{r}), \\
\text { where } \quad \varphi_{>}(\boldsymbol{r}) \geqq 0 \quad \text { and } \quad \varphi_{<}(\boldsymbol{r}) \leqq 0,
\end{gathered}
$$

as already introduced in Section II. The corresponding parts of the total cell-cell interactions, $\Phi_{N}^{+}(\boldsymbol{L})$ and $\Phi_{N}^{-}(\boldsymbol{L})$, are defined by replacing $\varphi$ in (3.7) by $\varphi_{>}$and $\varphi_{<}$, respectively.

Now consider the intermediate total interaction potential

$$
U_{N}^{+}\{\varphi\}=U_{N}\{\varphi\}+\Phi_{N}^{+}\{\boldsymbol{L} ; \varphi\} .
$$

Since the positive, or repulsive, interactions in $\Phi_{N}^{+}$can only reduce the configurational integral we clearly have

$$
Z(\beta, N, \Lambda) \geqq Z^{+}(\beta, N, \Lambda)
$$

where the superscript + denotes the use of the intermediate potential $U_{N}^{+}$. On the other hand (4.2) also implies

so that

$$
U_{N}^{+}\{\varphi\}=U_{N}^{\Pi}\{\varphi\}-\Phi_{N}^{-}\{\boldsymbol{L} ; \varphi\}
$$

$$
Z^{+}(\beta, N, \Lambda)=Z(\beta, N, \Pi)\left\langle\exp \left[+\beta \Phi_{N}^{-}(\boldsymbol{L})\right]\right\rangle_{I},
$$


where the angular brackets, $\langle\cdot\rangle$, denote the usual canonical thermal average while the subscript $\Pi$ indicates that the average is taken in the periodic system, i.e., with potential $U_{N}^{I I}$. By combining (4.3) and (4.5) and using the elementary inequality $\langle\exp (\cdot)\rangle \geqq \exp (\langle\cdot\rangle)$, we obtain the result

$$
g(\beta, \varrho ; \Pi) \leqq g(\beta, \varrho ; \Lambda)+\beta\left\langle-\Phi_{N}^{-}\right\rangle_{\Pi} / V(\Lambda) .
$$

In order to bound the last term in this inequality we split the total attractive cell-cell interaction, $\Phi_{N}^{-}=-\left|\Phi_{N}^{-}\right|$, into two parts:

(i) $\Psi_{N}^{<}(\boldsymbol{L}, R)$, consisting of all those interactions of particles in cell $\Lambda_{0}$ with those images of particles in the first shell of cells for which the particle-image distance satisfies $\left|\boldsymbol{r}_{j}+\boldsymbol{l}_{\boldsymbol{t}}-\boldsymbol{r}_{i}\right| \leqq R<\tilde{L}=L_{\min }$; and

(ii) $\Psi_{N}^{>}(\boldsymbol{L}, R)$, consisting of all the remaining cell-cell interaction terms for which the particle-image distance exceeds $R$.

Now the second or "long range" term can be bounded by using arguments precisely analogous to those used in Section III to bound $\Phi_{N}(\boldsymbol{L}, R)$ from above. Specifically, we use the partial stability condition $\mathbf{A}^{\prime}$ [Eq. (2.9)] and estimate the lattice sums as before [9]. In analogy to (3.10) and (3.11) we thence obtain

$$
\left|\psi_{N}^{>}(\boldsymbol{L}, R)\right| \leqq \frac{1}{2} N^{2} R^{-v} \psi^{-}(R),
$$

where $\psi^{-}(R)$ is continuous and vanishes as $\boldsymbol{L} \rightarrow \infty$ and is given explicitly by

$$
\psi^{-}(R)=\sum_{s=1}^{\infty}\left[\left(2+s^{-1}\right)^{v}-\left(2-s^{-1}\right)^{v}\right] \theta^{-}(s R) .
$$

To discuss the short range part of the interaction, $\Psi_{N}^{<}$, we first define the pair distribution function for the torus $\Pi$ by [13]

$$
\begin{aligned}
& n_{2}\left(\boldsymbol{r}_{1}, \boldsymbol{r}_{2} ; \beta, \varrho: \Pi\right) \\
& \quad=\frac{1}{(N-2) ! Q(\beta, N, \Pi)} \int d \boldsymbol{r}_{3} \ldots \int d \boldsymbol{r}_{N} \exp \left[-\beta U_{N}^{\Pi}\left(\boldsymbol{r}_{1}, \ldots \boldsymbol{r}_{N}\right)\right] .
\end{aligned}
$$

Notice that the translational invariance of the torus implies

$$
n_{2}\left(\boldsymbol{r}_{1}, \boldsymbol{r}_{2} ; \Pi\right) \equiv n_{2}\left(\boldsymbol{r}_{2}-\boldsymbol{r}_{1} ; \Pi\right)
$$

which is not, of course, true for the pair distribution function of the box $\Lambda$. Then from the definition

where

$$
\left\langle\Psi_{N}^{<}(\boldsymbol{L}, R)\right\rangle_{\Pi}=\frac{1}{2} \sum_{i=j}^{N} \sum_{j=1}^{N} \sum_{\boldsymbol{t} \neq \mathbf{0}}\left\langle\Theta_{R}\left(\left|\boldsymbol{r}_{i, j, \boldsymbol{t}}\right|\right) \varphi_{<}\left(\boldsymbol{r}_{i, j, \boldsymbol{t}}\right)\right\rangle_{\Pi}
$$

$$
r_{i, j, t}=r_{j}+l_{t}-r_{i}
$$


and

we obtain

$$
\begin{aligned}
& \Theta_{R}(r)=1 \text { for } r \leqq R, \\
& =0 \text { for } r>R \text {, }
\end{aligned}
$$

$$
\left\langle\left|\Psi_{N}^{<}(\boldsymbol{L}, R)\right|\right\rangle_{\Pi}=\frac{1}{2}\left(1-N^{-1}\right)^{-1} \int^{\prime} d \boldsymbol{r}_{1} \int_{|\boldsymbol{r}| \leqq R}^{\prime} d \boldsymbol{r}\left|\varphi_{<}(\boldsymbol{r})\right| n_{2}(\boldsymbol{r} ; \Pi) .
$$

Here the primes on the integrals indicate that the vector $\boldsymbol{r}_{2}=\boldsymbol{r}_{1}+\boldsymbol{r}$ must lie outside $\Lambda$ i.e., in a cell of the first shell. The maximum volume of integration for $\boldsymbol{r}_{1}$ is hence

$$
\begin{aligned}
V(\Delta)= & V(\Lambda)-\sum_{\alpha=1}^{v}\left(L_{\alpha}-2 R\right) \\
& \leqq 2 R \sum_{\alpha=1}^{v}\left[V(\Lambda) / L_{\alpha}\right] \leqq 2 v V(\Lambda) R / \tilde{L},
\end{aligned}
$$

where we assume $2 R<\tilde{L}=L_{\min }$. A simple bound may hence be obtained by restricting $\boldsymbol{r}_{1}$ to $\Delta$ but extending the integral over $\boldsymbol{r}$ to all space. This yields

where

$$
\beta\left\langle\left|\Psi_{N}^{<}\right|\right\rangle / V(\Lambda) \leqq 2 v \varrho^{2}\left\langle-\beta \varphi_{<}\right\rangle_{\Pi} R / \tilde{L}
$$

$$
\left\langle-\beta \varphi_{<}\right\rangle_{\Pi}=\beta \int d \boldsymbol{r}\left|\varphi_{<}(\boldsymbol{r})\right| n_{2}(\boldsymbol{r} ; \beta, \varrho ; \Pi),
$$

and where we have used $\left(1-N^{-1}\right)^{-1} \leqq 2$ for $N \geqq 2$.

To bound this last expectation value we consider the extended potentials $\varphi(\eta ; \boldsymbol{r})$ and $U_{N}^{\Pi}(\eta)$, introduced in Section II [Eqs. (2.18) to (2.20)]. An upper bound to the corresponding extended free energy density function now follows easily [14] from the extended periodic stability condition $\mathbf{A}^{\Pi} \boldsymbol{\eta}$ [Eq. (2.20)], namely,

$$
g(\eta ; \beta, \varrho ; \Pi) \leqq G^{+}=\varrho\left[1+\beta w_{A}\left(\eta_{0}\right)-\ln \left(\varrho \Lambda_{T}^{v}\right)\right] \text { for } \eta \leqq \eta_{0},
$$

where $\eta_{0}$ specifies the range over which the stability is guaranteed. This bound is uniformly valid for all $\Pi$ as $\boldsymbol{L} \rightarrow \infty$. A lower bound for the special value $\eta=0$ (which is all that will be needed) follows directly from the previous results (3.13) to (3.16) which we may write as

$$
g(\eta ; \beta, \varrho ; \Pi)_{\eta=0} \geqq G^{-}(\Pi) \rightarrow g_{\infty}(\beta, \varrho), \quad \text { as } \quad \boldsymbol{L} \rightarrow \infty,
$$

provided $\varrho$ is not too close to $\varrho_{\max }(\Lambda)$ or, asymptotically, provided $\varrho<\varrho_{\max }^{\infty}$.

Next we notice [13] that the desired expectation value (4.16) satisfies the identity

$$
\left\langle-\beta \varphi_{<}\right\rangle_{\Pi}=\left.(\partial / \partial \eta) g(\eta ; \beta, \varrho ; \Pi)\right|_{\eta=0},
$$

which, indeed, provides the rationale for introducing the extended potentials. Furthermore [13] by virtue of its definition as an exponential 
mean [via (1.2) and (1.7)] the function $g(\eta)$ is convex for $\eta \leqq \eta_{0}$, i.e.,

$$
g\left(\frac{1}{2} \eta_{1}+\frac{1}{2} \eta_{2}\right) \leqq \frac{1}{2} g\left(\eta_{1}\right)+\frac{1}{2} g\left(\eta_{2}\right), \quad\left(\eta \leqq \eta_{0}\right) .
$$

This means that the derivative $\partial g / \partial \eta$ at $\eta=0$ can be bounded in terms of $G^{+}$and $G^{-}$: specifically we obtain

$$
\left\langle-\beta \varphi_{<}\right\rangle_{\Pi} \leqq c_{2}=\left(G^{+}-G^{-}\right) / \eta_{0}<\infty,
$$

for $\varrho<\varrho_{\max }^{\infty}$ and large enough $\Lambda$.

On combining (4.7) with (4.15) and (4.21) and using again the shape condition $\mathbf{D}^{*}$ [Eq. (2.22)], the last term in the inequality (4.6) is seen to satisfy

$$
\beta\left\langle-\Phi_{N}^{-}\right\rangle_{\Pi} / V(\Lambda) \leqq \frac{1}{2} \beta \varrho^{2} c_{1}(\tilde{L} / R)^{v} \psi^{-}(R)+2 v \varrho^{2} c_{2}(R / \tilde{L}) .
$$

Then, in analogy with the previous analysis, we may choose $R$ such that

which implies

$$
R^{v} /\left[\psi^{-}(R)\right]^{1-\varepsilon}=\tilde{L}^{v}, \quad 1>\varepsilon>0,
$$

$$
\begin{aligned}
& (\tilde{L} / R)^{v} \psi^{-}(R) \rightarrow 0, \quad \text { and } \\
& R / \tilde{L} \rightarrow 0, \text { as } L \rightarrow \infty .
\end{aligned}
$$

Evidently with this choice of $R(\boldsymbol{L})$ we may take the limit $\boldsymbol{L} \rightarrow \infty$ in (4.6) to obtain the desired upper bound

$$
\limsup _{\boldsymbol{L} \rightarrow \infty} g(\beta, \varrho ; \Pi) \leqq g_{\infty}(\beta, \varrho) .
$$

In combination with the corresponding lower bound (3.5) this completes the proof of the main Theorem (1.12).

\section{Surface Terms}

It is natural to inquire more closely into the difference between the reduced free energy, $g(\Pi)$, for a torus and that for the corresponding box, namely $g(\Lambda)$, both at the same $\beta$ and $\varrho$. It is normally believed that this difference should amount to no more than a surface term; more specifically, one would like to prove that

$$
K^{+} S(\Lambda) / V(\Lambda) \geqq g(I)-g(\Lambda) \geqq-K^{-} S(\Lambda) / V(\Lambda),
$$

where the total surface "area" of $\Lambda$ is

$$
S(\Lambda)=2 \sum_{\gamma=1}^{\nu} S_{\gamma}, \quad S_{\gamma}=\sum_{\alpha \neq \gamma}^{\nu} L_{\alpha},
$$


and $\mathrm{K}^{+}$and $\mathrm{K}^{-}$are constants (depending on $\beta$ and $\varrho$ ). One also expects (5.1) to hold with either $g(\Pi)$ or $g(\Lambda)$ replaced by their thermodynamic limit $g_{\infty}(\beta, \varrho)$. A short examination reveals, however, that (5.1) should be expected to apply only when the pair interaction potentials decrease rather more rapidly as $r \rightarrow \infty$ than needed merely for stability and tempering. In essence, the existence of the first moment, $\int_{|\boldsymbol{r}|>\boldsymbol{r}_{0}}|\boldsymbol{r}| \varphi(\boldsymbol{r}) \mid \mathrm{d} \boldsymbol{r}$, seems to be necessary. To this end we will assume:

$\mathbf{S}$ The conditions $\mathbf{A}^{\prime}, \mathbf{B}^{\prime}, \mathbf{A} \mathbf{b}$ and $\mathbf{A}_{2}^{\Pi}$ (stated in Section II) hold with $\varphi(\boldsymbol{r})$ replaced by $\left(1+c_{0}|\boldsymbol{r}|\right) \varphi(\boldsymbol{r}) \quad\left(c_{0}>0\right)$ and with $\theta^{ \pm}(r)$ monotonic nonincreasing.

(Previously only $r^{-v} \theta^{ \pm}(r)$ was supposed monotonic.) Along the lines used in Section IV it is then not hard to prove the first inequality of (5.1). The second inequality is more difficult (as are the corresponding inequalities involving $g_{\infty}$ ) and we will utilize further conditions on our analysis although there seems little doubt that they are not necessary.

\section{Upper Bound}

To prove the first inequality we rewrite (4.6) with the decomposition $\Phi_{N}^{-}=\Psi_{N}^{<}+\Psi_{N}^{>}$as

$$
g(\Pi)-g(\Lambda) \leqq h^{<}+h^{>},
$$

where by the same arguments as lead to (4.7) and (4.8) we have

$$
h^{>}=\beta\left\langle\left|\Psi_{N}^{>}\right|\right\rangle_{\Pi} / V(\Lambda) \leqq \frac{1}{2} \beta \varrho\left(N / R^{v}\right) \psi_{S}^{-}(R)
$$

in which, using condition $\mathbf{S}$,

$$
\psi_{S}^{-}(R)=\sum_{s=1}^{\infty}\left[\left(2+s^{-1}\right)^{v}-\left(2-s^{-1}\right)^{v}\right] \theta^{-}(s R) /\left(1+c_{0} s R\right) .
$$

By removing the factor in brackets for $s=1$ and using the monotonicity of $\theta^{-}(r)$ to replace the sum by an integral we find

$$
\begin{aligned}
\xi^{-}(R)=\frac{1}{2} R \psi_{S}^{-}(R) & \leqq\left(3^{v}-1\right) \int_{\frac{1}{2} R}^{\infty} d r \theta^{-}(r) /\left(1+c_{0} r\right), \\
& \rightarrow 0, \text { as } R \rightarrow \infty,
\end{aligned}
$$

where the second line follows from the integrability of $r^{-1} \theta^{-}(r)$. On combining (5.4) to (5.6), choosing $R=\tilde{L}$, and using the shape condition D* $^{*}$ of Section II we obtain

$$
h^{>} \leqq c_{1} \beta \varrho^{2} \xi^{-}(\tilde{L}) / \tilde{L} \leqq c_{1}^{2} \beta \varrho^{2} \xi^{-}(\tilde{L})\left[\tilde{L}^{v-1} / V(\Lambda)\right] .
$$


For the short range part, $h^{<}$, we use (4.13) with $R=\tilde{L}$ and find, for $N \geqq 2$,

$$
H^{<}=[V(\Lambda) / \beta] h^{<} \leqq \int_{\Lambda}^{\prime} d \boldsymbol{r}_{1}^{\prime} \int_{|\boldsymbol{r}| \leqq L}^{\prime} d \boldsymbol{r}\left|\varphi_{<}(\boldsymbol{r})\right| n_{2}(\boldsymbol{r} ; \Pi),
$$

where the primes indicate that $\boldsymbol{r}^{\prime \prime}=\boldsymbol{r}+\boldsymbol{r}^{\prime}$ must lie outside $\Lambda$, and $n_{2}(\boldsymbol{r} ; \Pi)$ is the pair correlation function for the torus [defined in (4.9)]. The integrand in (5.8) is non-negative for all $\boldsymbol{r}$ so that extending the range of integration, and integrating more than once over some regions can only strengthen the inequality. To perform the $\boldsymbol{r}^{\prime}$ integration we thus take in turn an origin in each face of the pairs $\gamma=1,2, \ldots v$ with a sense such that $r_{\gamma}^{\prime}=\left(\boldsymbol{r}^{\prime}\right)_{\gamma}>0$ for points inside $\Lambda$. Then we obtain the bound

$$
H^{<} \leqq \sum_{\gamma=1}^{v} S_{\gamma} \int_{0}^{\tilde{L}} d r_{\gamma}^{\prime}\left(\int_{\boldsymbol{r}_{\gamma} \geqq \boldsymbol{r}_{\gamma}^{\prime}} d \boldsymbol{r}+\int_{\boldsymbol{r}_{\gamma} \leqq-\boldsymbol{r}_{\gamma}^{\prime}} d \boldsymbol{r}\right)\left|\varphi_{<}(\boldsymbol{r})\right| n_{2}(\boldsymbol{r} ; \Pi) .
$$

On interchanging the order of integration the limits become

$$
\left(\int_{r_{\gamma} \geqq 0} d \boldsymbol{r}+\int_{r_{\gamma} \leqq 0} d \boldsymbol{r}\right)^{\min \left\{\tilde{L}, r_{\gamma}\right\}} \int_{0} d r_{\gamma}^{\prime} .
$$

The inequality is again strengthened if the limit on the $r_{\gamma}^{\prime}$ integration is now extended to $r_{\gamma}$ (for all $\tilde{L}$ ). This yields

$$
H^{<} \leqq c_{1} \tilde{L}^{\nu-1} \int d \boldsymbol{r}\left(\sum_{\gamma}\left|r_{\gamma}\right|\right)\left|\varphi_{<}(\boldsymbol{r})\right| n_{2}(\boldsymbol{r} ; \Pi),
$$

where we have used condition $\mathbf{D}^{*}$ through

$$
S_{\gamma}=V(\Lambda) / L_{\gamma} \leqq c_{1} \tilde{L}^{\nu-1} .
$$

On noting that $\left|r_{\gamma}\right| \leqq|\boldsymbol{r}|$, and adding a further nonnegative term proportional to $\left|\varphi_{<}(\boldsymbol{r})\right|$ we find

$$
H^{<} \leqq\left(v c_{1} / c_{0}\right) \tilde{L}^{v-1} \int d \boldsymbol{r}\left(1+c_{0}|\boldsymbol{r}|\right) n_{2}(\boldsymbol{r} ; \Pi) .
$$

The integral here can evidently be expressed as $\left\langle-\left(1+c_{0}|\boldsymbol{r}|\right) \varphi_{<}(\boldsymbol{r})\right\rangle_{\Pi}$ and hence may be bounded by exactly the same convexity arguments that led to (4.21), in which $c_{2}$ was defined. We conclude, therefore, that

$$
h^{<} \leqq\left(v c_{1} c_{2} / c_{0}\right) \tilde{L}^{v-1} / V(\Lambda) \text {. }
$$

Finally, if we notice from (5.2) that

$$
2 v \tilde{L}^{v-1} \leqq S(\Lambda),
$$

and combine (5.14) and (5.7) in (5.3), we see that the first part of (5.1) is established (for $\tilde{L}>R_{0}$ ) with

$$
K^{+}=\frac{1}{2}\left(c_{1} c_{2} / c_{0}\right)+\frac{1}{2} c_{1}^{2} \beta \varrho^{2} \xi^{-}\left(R_{0}\right) / v .
$$




\section{Lower Bound}

The idea we use here is first to expand the torus $\Pi$ to a torus $\Pi^{+}=\omega \Pi$ corresponding to an enlarged box $\Lambda^{+}$of sides $L_{\alpha}^{+}=\omega L_{\alpha}(\omega \geqq 1)$; secondly, to insert corridors of width exceeding $R=(\omega-1) \tilde{L}$, as in Section III, so as to reduce the torus $\Pi^{+}$to the original box $\Lambda$. The effects of then discarding the positive cell-cell interactions must be shown to be bounded by a surface term. This last step is not hard if the potentials have a hard core $[\varphi(\boldsymbol{r})=+\infty$ for $r<a]$ so that the density has a bound $\varrho_{\max }$; without the aid of a hard core, however, we have been unable to complete the argument. Conversely, if we assume a hard core, the problem of bounding the free energy change on the expansion $\Pi \rightarrow \Pi^{+}$has so far proved intractable.

Accordingly, we will assume the pair potential is strongly tempered, i.e., it satisfies condition $\mathbf{B}^{*}$ [see Eq. (3.1)]. The second step is then trivial for $R \geqq R_{0}$. To facilitate the first step we introduce the extended stability condition:

$\mathbf{S}^{\Pi !} \boldsymbol{\zeta}$ Periodic Surface Stability

(i) $\varphi(\boldsymbol{r})$ is differentiable for all $\boldsymbol{r} \neq \mathbf{0}$;

(ii) for all $\boldsymbol{L}$ with $\tilde{L} \geqq R_{1}>0$

$$
\sum_{\boldsymbol{t} \neq 0} \boldsymbol{l}_{\boldsymbol{t}} \cdot \nabla \varphi\left(\boldsymbol{l}_{\boldsymbol{t}}\right) \geqq-v_{0},
$$

(iii) for some $\zeta_{0}>0$ and all $N$

$$
\begin{aligned}
& U_{N}^{I}(\zeta)=U_{N}^{I I}\left\{\varphi(\boldsymbol{r})+\zeta \boldsymbol{r} \cdot \nabla \varphi(\boldsymbol{r}) \geqq-N v_{A}(\zeta),\right. \\
& \text { with } v_{A}(\zeta)<\infty \text { for } \zeta \leqq \zeta_{0} .
\end{aligned}
$$

The last condition, (iii), namely, periodic stability, will be satisfied, as before, if $\varphi+\zeta \boldsymbol{r} \cdot \nabla \varphi$ satisfies condition $\mathbf{A b}$, i.e., is of positive type plus a positive potential. Although this condition allows suitable power law potentials [1-3] diverging like $1 / r^{\mu}$, it definitely excludes potentials with a hard core. The differentiability condition (i) could be relaxed somewhat at the cost of more elaborate analysis.

We now present the line of argument. For convenience we set

$$
f_{N}(\beta ; \Pi)=\varrho^{-1} g(\beta, \varrho ; \Pi)=N^{-1} \ln Z(\beta, N, \Pi),
$$

and consider the derivative

$$
j(\omega)=(\partial / \partial \omega) f_{N}(\beta ; \omega \Pi) .
$$

We will establish the bound

$$
j(\omega) \leqq J \text { for } 1 \leqq \omega \leqq \omega_{0},
$$


which, on integration, leads to

$$
f_{N}\left(\beta, \Pi^{+}\right) \leqq f_{N}(\beta ; \Pi)+J(\omega-1), \quad\left(\omega \leqq \omega_{0}\right) .
$$

Now choose $\omega$ according to

$$
\omega=1+(R / \tilde{L}) \text { with } R \geqq R_{0},
$$

where $R$ is the fixed width of the narrowest of the channels to be inserted in order to reduce $\Pi^{+}$to $\Lambda$. On using $\mathbf{B}^{*}$ to discard the residual cell-cell interactions we have

$$
f_{N}\left(\beta, \Pi^{+}\right) \geqq f_{N}(\beta, \Lambda) .
$$

Combining this with (5.22) yields, for large enough $\tilde{L}$,

$$
g(I)-g(\Lambda) \geqq-J \varrho R / \tilde{L},
$$

which reduces to the desired lower bound of (5.1), with

$$
K^{-}=J \varrho c_{1} R / 2 v,
$$

when (5.12) and (5.15) are used.

\section{Approach to Thermodynamic Limit}

Before proving the bound (5.21) we note that we may equally consider the sequence of tori $k \Pi(k=1,2,3, \ldots)$ in place of $\Pi$, and divide up each $k \Pi^{+}=\omega k \Pi$ by channels into $k$ replicas of the box $\Lambda$ with $N$ particles in each box. On using the basic inequality [1]

$$
Z\left(\beta, k N, k \Pi^{+}\right) \geqq[Z(\beta, N, \Lambda)]^{k}
$$

and using (5.22) for $k \Pi$ we find

$$
f_{k N}(\beta, k \Pi) \geqq f_{N}(\beta, \Lambda)-J(R / \tilde{L}) .
$$

If we let $k \rightarrow \infty$ and use the previously proved result, namely $g(k \Pi) \rightarrow g_{\infty}$, we obtain in place of (5.25) the additional result

$$
g(\beta, \varrho ; \Lambda)-g_{\infty}(\beta, \varrho) \leqq K^{-} S(\Lambda) / V(\Lambda) .
$$

In combination with the upper bound (5.1), already established, this yields

$$
g(\beta, \varrho ; \Pi)-g_{\infty}(\beta, \varrho) \leqq K_{1}^{-} S(\Lambda) / V(\Lambda)
$$

where $K_{1}^{-}=K^{-}+K^{+}$. These results give information on the rapidity of approach to the thermodynamic limit from above. Unfortunately we have not so far been able to establish corresponding bounds on the approach to the limit from below. 
It remains to justify the bound (5.21). The derivative $j(\omega)$ may be calculated, as in the usual formal derivations of the "virial theorem", by changing integration variables in the partition function from $\boldsymbol{r}_{i}$ to $\boldsymbol{r}_{i}^{\prime}=\boldsymbol{r}_{i} / \omega$ before differentiation. Using the expressions (2.4) to (2.6) for $U_{N}^{I}$ then leads to

where

$$
\begin{aligned}
j(\omega)=v & -\frac{1}{2} \beta \sum_{\boldsymbol{t} \neq 0} \boldsymbol{l}_{\boldsymbol{t}} \cdot \nabla \varphi\left(\boldsymbol{l}_{t}\right) \\
& -\frac{1}{2}[V(\omega \Pi) / N] \beta \int_{\Lambda} d \boldsymbol{r}\left[\sum_{\boldsymbol{t}} \boldsymbol{r}_{\boldsymbol{t}} \cdot \nabla \varphi\left(\boldsymbol{r}_{\boldsymbol{t}}\right)\right] n_{2}(\boldsymbol{r} ; \omega \Pi)
\end{aligned}
$$

$$
\boldsymbol{r}_{\boldsymbol{t}}=\boldsymbol{r}+\boldsymbol{l}_{\boldsymbol{t}}
$$

and, $n_{2}(\boldsymbol{r} ; \omega \Pi)$ is the (translationally invariant) pair correlation function for the torus $\omega \Pi$. On using part (ii) of condition $\mathbf{S}^{\Pi} \boldsymbol{\zeta}$ this leads along the previous lines to

$$
j(\omega) \leqq v+\frac{1}{2} \beta v_{0}+\frac{1}{2} \omega_{0}^{v} \varrho^{-1}\langle-\beta \boldsymbol{r} \cdot \nabla \varphi(\boldsymbol{r})\rangle_{\omega \Pi}
$$

for $1 \leqq \omega \leqq \omega_{0}$ where, in terms of the extended potential $U_{N}^{\Pi}(\zeta)$ of $(5.18)$, we have

$$
\langle-\beta \boldsymbol{r} \cdot \nabla \varphi(\boldsymbol{r})\rangle_{\omega \Pi}=\left.(\partial / \partial \zeta) g(\zeta ; \beta, \varrho ; \omega \Pi)\right|_{\zeta=0} .
$$

Now we can use the stability and convexity argument [13] of Eqs. (4.17) to (4.21) once again to prove

$$
\left(\partial g / \partial \zeta_{\zeta=0} \leqq c_{3}, \quad\left(1 \leqq \omega \leqq \omega_{0}\right),\right.
$$

where in terms of the bounds $G_{\zeta}^{+} \geqq g\left(\zeta_{0}\right)$ [replace $w_{A}\left(\eta_{0}\right)$ in $(4.17)$ by $\left.v_{A}\left(\zeta_{0}\right)\right]$, and $G^{-} \leqq g(\zeta=0)$ [see Eqs. (3.13) to (3.16)], we have $c_{3}=\left(G_{\zeta}^{+}-G^{-}\right) / \zeta_{0}$. Combination of these last three relations proves (5.21) and completes our analysis.

\section{Grand Canonical Ensemble}

All our previous analysis has referred to the canonical ensemble. Although we have not made a detailed investigation, we believe our arguments will extend without significant difficulty to the microcanonical ensemble since the geometrical problems associated with fixed particle number are similar in that context. On the other hand, most of the analysis becomes rather simpler in the grand canonical ensemble and analogs of all our results may be proved. Furthermore, we can then obtain a surface-to-volume lower bound, analogous to (5.1), with the presence of a hard core in the potential; indeed, the hard core enables us 
to analyze, in addition, weakly tempered potentials (positive at large $r$ ). It is also possible to prove that the grand canonical pressure approaches its thermodynamic limit at the expected rate. We now sketch the arguments for this case but omit the detail (most of which is similar to that presented already).

The grand canonical pressure $p(\beta, z ; \Lambda)$ is related to the grand canonical partition function

as usual, by

$$
\Xi(\beta, z ; \Lambda)=\sum_{N=1}^{\infty} z^{N} Z(\beta, N, \Omega),
$$

$$
\Xi(\beta, z ; \Lambda)=\exp [V(\Lambda) \beta p(\beta, z ; \Lambda)],
$$

with similar expressions for the torus $\Pi$.

Now the existence of a hard core, and hence a maximum density $\varrho_{\max }$, together with condition $\mathbf{S}$ of the previous section (or merely $\mathbf{A}^{\prime}$ ) means that the energy of interaction of a single particle with all others is bounded below by some $-w_{0}\left(w_{0}<\infty\right)$. By sqeezing the box $\Lambda$ to a smaller box $\Lambda^{-}$, which can be done by "switching-off" the interactions of particles in the channels $\Delta=\Lambda-\Lambda^{-}$, we see that

$$
\Xi(\beta, z ; \Lambda) \leqq \Xi\left(\beta, z ; \Lambda^{-}\right) e^{z V(\Delta)} e^{\mathcal{N}(\Delta) \beta w_{0}},
$$

where $\mathcal{N}(\Delta)$ is the maximum number of particles that can be accommodated in $\Delta$. (It is the analog of this formula that we could not obtain for the canonical case; the difficulty was that the (fixed) number of particles in $\Lambda$ had all to go into $\Lambda^{-}$whereas here the population in $\Lambda^{-}$can vary.) Now we have

$$
\mathscr{N}(\Delta) \leqq \varrho_{\max } V(\Delta)
$$

and, for fixed channel width $R$ and large enough $\tilde{L}$, also

$$
V(\Delta) \leqq c_{4} R S(\Lambda) \text {. }
$$

Of course we choose $R$ to exceed the hard core diameter $a$. Then we may compare the torus with $\Lambda^{-}$which yields, term by term,

$$
\Xi(\beta, z ; \Pi) \geqq \sum_{N=0}^{\infty} z^{N} Z\left(\beta ; N, \Lambda^{-}\right) \exp \left[-\beta \max \left\{\Phi_{N}^{+}\right\}\right] .
$$

To bound $\Phi_{N}^{+}(R)$, the positive cell-cell interaction energy lost in reducing the torus to $\Lambda^{-}$, we utilize the bound on the density and consider the interactions across the $2 v$ faces of $\Lambda$ along the lines used in deriving (5.14) from (5.8). In particular, we utilize the upper bound of condition $\mathbf{S}$ and find

$$
\max \left\{\Phi_{N}^{+}\right\} \leqq N c_{5} \varrho_{\max } a^{v} w_{0} R S(\Lambda) / V(\Lambda)=N \varepsilon(\Lambda)
$$


for fixed $R$ and sufficiently large $\tilde{L}$. (The factor $a^{v} w_{0} R$ is inserted purely for dimensional reasons.) Hence (6.6) can be written

where

$$
\Xi(\beta, z ; \Pi) \geqq \Xi\left(\beta, z^{-}, \Lambda^{-}\right)
$$

$$
z^{-}=z e^{-\beta \varepsilon(\Lambda)} .
$$

On combining this result with Eqs. (6.3) to (6.5) we obtain

$$
p(\beta, z ; \Pi) \geqq p\left(\beta, z^{-} ; \Lambda\right)-c_{4} R\left(k_{\mathrm{B}} T z+\varrho_{\max } w_{0}\right) S(\Lambda) / V(\Lambda) .
$$

Lastly, we may use the convexity and boundedness of $p(z)$ in $\ln z$ to prove that

$$
p(\beta, z ; \Lambda)-p\left(\beta, z^{-} ; \Lambda\right) \leqq c_{6} \varrho_{\max } k_{\mathrm{B}} T\left[\ln z-\ln z^{-}\right],
$$

where the factor $\varrho_{\max } k_{\mathrm{B}} T$ is again retained for dimensional reasons. Together with (6.9) and (6.7) we hence obtain the desired bound

with

$$
p(\beta, z ; \Pi)-p(\beta, z ; \Lambda) \geqq-E^{-} S(\Lambda) / V(\Lambda)
$$

$$
E^{-}=\left[c_{5} c_{6}\left(\varrho_{\max }\right)^{2} a^{v} w_{0}+c_{4}\left(k_{\mathrm{B}} T z+\varrho_{\max } w_{0}\right)\right] R .
$$

We recall that this result was established under the assumptions that the potential has a hard core although $r \varphi(\boldsymbol{r})$ (see $\mathbf{S})$ need only satisfy the mild tempering condition $\mathbf{B}^{\prime}$.

To discuss the approach to the thermodynamic limit we note that the inequality (6.3) holds for a box $\Lambda_{k}=k \Lambda$ [i.e., $\boldsymbol{L}_{k}=k \boldsymbol{L}$ ] with the left member replaced by $[\Xi(\beta, z ; k \Lambda)]^{1 / k}$; one simply inserts sufficiently many channels to decompose $\Lambda_{k}$ into $k$ replicas of $\Lambda^{-}$, and notes the estimates $V(k \Delta) \approx k V(\Delta)$ and $\mathscr{N}(k \Delta) \approx k \mathcal{N}(\Delta)$. On letting $k \rightarrow \infty$ and using the existence of the grand canonical thermodynamic limit, namely,

$$
\lim _{k \rightarrow \infty} p(\beta, z ; k \Lambda)=p_{\infty}(\beta, z),
$$

one obtains, in place of (6.12), the result

$$
p(\beta, z ; \Pi)-p_{\infty}(\beta, z) \geqq-E^{-} S(\Lambda) / V(\Lambda) .
$$

Essentially the same argument yields the inequality with the box pressure $p(\beta, z ; \Lambda)$ replacing the torus pressure $p(\beta, z ; \Pi)$. These inequalities are the analogs of those which were not proved for the canonical ensemble. [Compare with Eqs. (5.29) and (5.30).] On combining them with the corresponding inequalities for the approach to the thermodynamic limit from above we see that for large boxes or tori the grand canonical pressure differs from its limit by no more than a surface-to-volume term.

In conclusion we note that Ruelle [15] has very recently shown that for superstable potentials (i.e., $\varphi(\boldsymbol{r})$ is the sum of a stable potential and 
a positive continuous function $\psi(\boldsymbol{r})$ with $\psi(\mathbf{0})>0)$ the grand canonical correlation functions can be uniformly bounded. This should open the way to further progress for the grand ensemble. Likewise, when a similar result becomes available for canonical correlation functions it should be possible to extend our results appreciably.

Acknowledgements. Our work on this problem was stimulated by our attendance at the very informal symposium on exact results in statistical mechanics held at the University of California at Irvine in November, 1968. We are gratefull to the organizers of this meeting, Professors Alexei Maradudin, Meinhard Mayer, and David Ruelle, for having invited us. We are indebted to Dr. G. Gallavotti and Professor David Ruelle for conversations and for comments on a draft of the manuscript. The support of the National Science Foundation, of the Advanced Research Projects Agency through the Materials Science Center at Cornell University, and of the United States Air Force Office of Scientific Research under Grant No. $68-1416 \mathrm{~B}$ is gratefully acknowledged.

\section{References}

1. Fisher, M. E.: Arch. Rat. Mech. Anal. 17, 377 (1964).

2. Ruelle, D.: Helv. Phys. Acta 36, 183, 789 (1963).

3. - Statistical mechanics - rigorous results. New York: W. A. Benjamin, Inc. 1969.

4. Lebowitz, J. L., Lieb, E. H.: Phys. Rev. Letters 22, 631 (1969).

5. Griffiths, R. B.: Phys. Rev. 176, 655 (1968).

6. See e.g., the dimer problem on a square lattice: Fisher, M. E.: Phys. Rev. 124, 1664 (1961) and Ferdinand, A. E.: J. Math. Phys. 8, 2332 (1967).

7. This proposition (due to D. Ruelle) has been proved in: Landford, O.: Commun. Math. Phys. 11, 257 (1969).

8. Fisher, M. E., Ruelle, D.: J. Math. Phys. 7, 260 (1966).

9. Lebowitz, J. L., Penrose, O.: J. Math. Phys. 7, 98 (1966), Eq. (2.8) et seq.

10. Lieb, E.: J. Math. Phys. 7, 1016 (1966).

11. Robinson, D. W.: Statistical mechanics of quantum mechanical particles with hard cores. I. The thermodynamic pressure. (C.N.R.S. Marseille, preprint 1970).

12. Fano, G., Galavotti, G., Lupini, L.: On the boundary conditions in quantum statistical mechanics, (preprint 1970).

13. See e.g., Fisher, M. E.: J. Math. Phys. 6, 1643 (1965).

14. See e.g., Ref. 1, Section IV.

15. Ruelle, D.: Superstable interactions in classical statistical mechanics (I.H.E.S. preprint 1970).

J. L. Lebowitz

Belfer Graduate School of Science

New York, N. J. 10033, USA
M. E. Fisher

Baker Laboratory, Cornell University Ithaca, New York 14850, USA 